\title{
Emotions in Collaborative Networks: A Monitoring System
}

\author{
Filipa Ferrada ${ }^{1}$ and Luis M. Camarinha-Matos ${ }^{1,2}$ \\ ${ }^{1}$ CTS, Uninova, Faculdade de Ciências e Tecnologia, Universidade Nova de Lisboa, \\ 2829-518 Caparica, Portugal \\ ${ }^{2}$ Faculdade de Ciências e Tecnologia, Universidade Nova de Lisboa, \\ 2829-518 Caparica, Portugal \\ \{faf, cam\} @uninova.pt
}

\begin{abstract}
In the last years the rapid progress of ICT along with the growing business models have led to the emergence of a large variety of collaborative networks. Collaboration brought new achievements such as the capacity of the involved entities getting into business in a sustainable and stable way. Despite the fact that collaboration has significant benefits, especially in creating value and boosting innovation, some studies indicate that most of them tend to fail. Among others, the lack of specific systems to monitor the emotional state of the network is one of the reasons why collaboration fails. Emotions can interfere in collaboration environments by increasing or decreasing the motivation and performance level and even in conflict resolutions. Therefore, it becomes particularly necessary to find means for supervising the emotional state of collaborative networks in order to achieve the equilibrium for a healthy community. This paper introduces an approach to develop an emotions-oriented system and presents two scenario cases of collaborative networks where this system can be implemented.
\end{abstract}

Keywords: Emotions, Collaborative Networks, Emotional Health, Collaborative Emotions, Emotions-oriented System.

\section{Introduction}

In recent years the emergence of a large variety of collaborative networks has become a reality. This is due to wide availability and rapid evolution of information and communication technologies along with the rapidly evolving challenges faced by business entities and the society in general [1].

Through collaboration, the involved entities can achieve their common goals and increase their own stability and sustainability, within the context of today's global economy, by sharing resources, sharing and exchanging information, reducing risks, costs, time-to-market, and delivery-time, and increasing the market share, assets utilization, skills and knowledge sharing, and customer services [1-5].

Emotions constitute a central element for human beings, especially in human interactions. Likewise, they can influence the experience of partners in collaboration environments by increasing the achievement and performance level, motivation, 
excitement in interaction with each other and the whole perception among individuals. Moreover, emotions can control the motivation for achieving goals as well as improving an individual's problem solving ability [6]. In this way, the study of their functioning is a prerequisite for the understanding of individual and collective behaviors [7-9].

In the perspective of collaborative networks $(\mathrm{CN})$, individual participants experience emotions not only as a result of directly experiencing events (that involve/evoke their own particular emotions) but also through collaborative interactions and by identifying themselves with the network as a whole. As a consequence, and viewing the collaborative network as a living body, it is dependent of the intricate interactions between the various participants, so it develops a (collective) emotion which is influenced by each individual participant's dominant emotions representing, in this way, the emotional state or the emotional climate of the network [10].

In this context, the introduction of a kind of supervision system to manage and monitor the emotional climate/state - or the "collective virtual emotions" - of collaborative networks with the intention of maintaining the emotional equilibrium of the community, forecasting and attempting to heal potential conflicts among participants and external communities are important elements for the success of the network. As Parung \& Bititci [5] claim, the healthier collaboration will have a longer life than less healthy ones.

This paper introduces an approach to develop and emotions-oriented system aimed at assisting in the management of the interaction among $\mathrm{CN}$ participants and the emotional dynamics that affect its normal operation, through the identification of possible emotional conflicts that may lead to serious problems. Two different types of CNs are presented and characterized and possible emotional conflicts for each $\mathrm{CN}$ are identified. The reminder of this paper is organized as follows: Section 2 identifies the contribution to value creation that this topic brings; Section 3 gives a brief description of how emotions can bring benefits to CNs; Section 4 presents the proposed emotions-oriented monitoring system; Section 5 presents the two collaborative networks cases. Finally Section 6 discusses the related research and Section 7 concludes.

\section{Contribution to Value Creation}

The process of value creation within collaborative networks is per se a great challenge, since it implies a fair co-creation and benefits distribution for the involved participants, including the customers. This passes through having a good management of the interactions among participants and the inherent emotional associated issues between them. Emotional conflicts between participants are not easily perceived and most of the times they generate direct negative impacts when co-creating, due to the fact that emotions are dynamic and uncontrollable. Especially when there is a disconnection between two partners - helping them connect may become time and costly consuming. The work presented at this paper aims at contributing in the process of value creation through contributing to a healthier exchange of information and communication among partners, with the introduction of the emotions assessment; creating avoidance of potential complicated conflicts, that sometimes are 
difficult to solve, in the co-creation processes; contributing to the collaboration technology, through the development of an emotional health monitoring tool including a set of mediation functionalities.

\section{Emotions in Collaborative Networks}

Despite the fact that collaboration has significant potential benefits especially in creating value and boosting innovation, some empirical studies also indicate that although the number of CNs is increasing, a large number fail [3]. Conducted research has identified several reasons for this high failure rate namely the lack of commitment and sharing goals among participants, lack of mutual trust, lack of agreed practices and values, difficulties in participants' relationships, participants' dissatisfaction with the collaboration outcome, or internal conflicts [3, 5, 11-13]. Furthermore, the efficiency and effectiveness of the decisions and activities within the network will depend on how good the interaction among partners is.

A new challenge emerges when dealing with "collective virtual emotions" within collaborative networks. In fact, several studies reveal that emotions are very often the cause for misunderstandings and conflicts which, in some cases might lead to the failure of collaborative networks. If we take for instance, the business negotiation example where different participants get together to negotiate the business propositions including delicate issues such as the risk sharing or profit distribution, it is likely that some tension and conflicts might arise. If a proper system could monitor the negotiation process, negative emotions found in the negotiation interaction among partners could be perceived and some assistance could be launched for the sake of the business and the network success. In this way, monitoring the interactions within the network might provide multi-modal emotional input (such as specific interfaces or special sensors borrowed from the Human-Computer Interaction research areas, e.g. from affective computing area [14]) for achieving awareness of each participant itself as well as the collective emotional state. Having these challenges been taken into consideration, the main research question chosen for this work is the following:

What could be a suitable set of models, methods and tools to promote emotional health in collaborative networks, namely allowing the diagnosis of the networks' emotional state and assisting in conflicts resolution?

The proposed hypothesis to address this research question is:

Collaborative networks' emotional health can be identified, modeled and promoted if a combination of contributions from human related sciences such as psychology, physiology and sociology along with human-computer interaction techniques and intelligent supervision systems, is used to develop models and methods which will serve as a basis for the design and development of an emotions-oriented supervision system focused on monitoring the emotional state of the network and providing emotions' selfregulation mechanisms. 
An illustration of the contributing mentioned research areas is presented in Fig. 1.

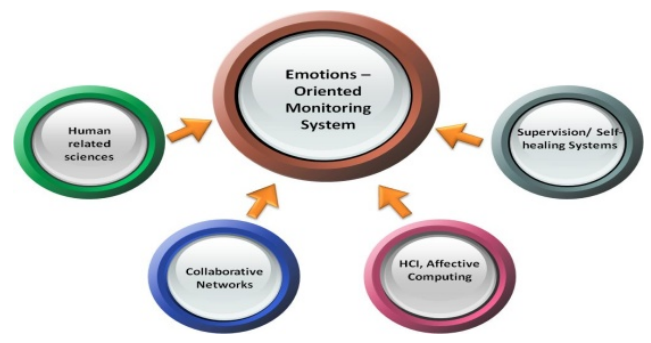

Fig. 1. Contributing research areas for an emotions-oriented system

\section{Emotions-Oriented Supervision System}

Supervising the emotional interactions within the collaborative network will provide multi-modal emotional input for achieving awareness of the participants as well as the collective emotional state.

The supervision system should offer mechanisms to perceive what is not emotionally working and, with or without the $\mathrm{CN}$ manager intervention, make the necessary adjustments to promote the emotional health of the $\mathrm{CN}$. It will comprise the following main modules, as illustrated in Fig. 2:

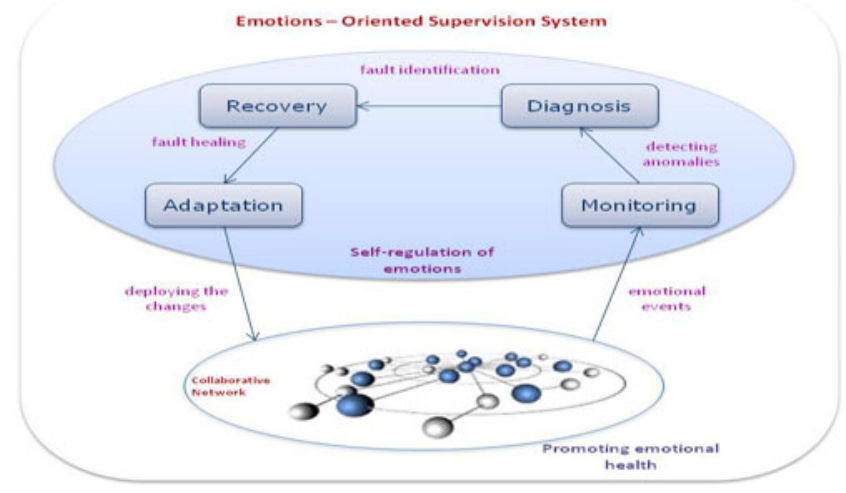

Fig. 2. Proposed emotions-oriented supervision system

- Monitoring. This module is in charge of scrutinizing all the participants' emotional interactions, seeking for anomalies. These malfunctions might occur when participants are socializing, negotiating a new business opportunity or when co-working in a project. 
- Diagnosis. After detecting that something is not properly working, this module tries a first diagnosis of the occurrence, identifying the emotional fault.

- Recovery. Once discovered the emotional problem, a healing process runs with the aim to restore the emotional state of the $\mathrm{CN}$ to normalcy.

- Adaptation. Finally this module is responsible to adapt itself by deploying the changes to the $\mathrm{CN}$.

The involved research areas in this supervision system include emotions related sciences, human-computer interactions and affective computing, self-healing systems and collaborative networks. Table 1 depicts the current analysis and characterization of the different contributing research areas.

Furthermore, emotional intelligence in appraising and evaluating the interaction with respect to the overall benefits for the $\mathrm{CN}$ opens the way for devising effective pro-active mediation mechanisms. In addition, as effective mediation is only possible through proper communication with partners, both affective approaches and social protocols are being taken into consideration for effective mediation in collaborative networks. Therefore, affective interaction protocols are being specified, extending the notion of social interaction protocol, and take into account the wider spectrum of emotion, mood and behavior, of both the participants and the $\mathrm{CN}$ as a whole.

Table 1. Analysis and characterization of the different contributing research areas.

\begin{tabular}{|c|c|c|c|}
\hline \multirow{3}{*}{$\begin{array}{l}\text { Emotions } \\
\text { related } \\
\text { sciences }\end{array}$} & $\begin{array}{l}\text { Social } \\
\text { psychology }\end{array}$ & \multicolumn{2}{|c|}{$\begin{array}{l}\text { Creation of proper emotional interaction models based on: } \\
\text { - The differential distribution of emotion across CN participants; } \\
\text { - Collective emotion; } \\
\text { - Emotion directed at other groups } \\
\text { - Role-related implications of emotional experience in collaboration contexts }\end{array}$} \\
\hline & \multirow{2}{*}{$\begin{array}{l}\text { Sociology } \\
\text { of emotions }\end{array}$} & $\begin{array}{l}\text { Collective \& Group- } \\
\text { based emotions }\end{array}$ & $\begin{array}{l}\text { Creation and design of models and methods to identify } \\
\text { and measure collective emotions within CNs. }\end{array}$ \\
\hline & & Social Work & $\begin{array}{l}\text { Concepts like emotions at work, emotion labor or work } \\
\text { and emotional intelligence help in identifying and } \\
\text { creating the emotional models for CNs. }\end{array}$ \\
\hline $\begin{array}{l}\text { Affective } \\
\text { Computing }\end{array}$ & \multicolumn{3}{|c|}{ Contribute as sensorial input for this work. } \\
\hline $\begin{array}{l}\text { Self-Healing } \\
\text { Systems }\end{array}$ & \multicolumn{3}{|c|}{$\begin{array}{l}\text { Contribute to the emotions-oriented supervision system mechanism envisioned in this work: } \\
\text { - Differentiate between the emotional "healthy" and "unhealthy" states; } \\
\text { - Assessment of the } \mathrm{CN} \text { manager intervention in the recovery process. } \\
\text { appended to the supervision systems (intelligent adaptation of machine learning } \\
\text { mechanisms or intelligent multi-agent systems). }\end{array}$} \\
\hline
\end{tabular}

In this way, the adopted approach is envisioned to comprise the following main blocks: (i) Models - building a conceptual framework aimed at developing a working definition of the involved concepts and their relationships; building formal models of emotions and emotional interactions within the context of collaborative networks. 
(ii) Methods and Mechanisms - elaboration of methods for perceiving and classifying collective emotions; identification of the main emotion-related functionalities within CNs; elaboration of emotional proactive mediation mechanisms. (iii) Prototype Tool Development of a software tool that supports the emotions-oriented supervision system integrating the identified functionalities and focusing on supporting the emotional health of the $\mathrm{CN}$.

\section{$5 \quad$ Case Scenarios}

In order to validate the proposed work, two case scenarios are presented. The first case represents a networked community of senior professionals while the second consists of a network of small companies' specialists in the solar parks construction.

\section{Seniors' Professional Network}

A network of senior professionals constitutes an association of senior professional individuals sharing their main compatible and/or common goals of increasing their active professional life in the society and/or market, through co-working with others in teams supported by computer networks. Their effective involvement in the socioeconomic system needs to consider other stakeholders, namely intermediary entities and the recipients of the services provided by seniors. Some of these entities are also organized in some form of association e.g. associations of SMEs, enterprise incubators or science parks, networks of regional development agencies and associated private (small) consultancy companies.

Understanding the dynamics of the collective emotions generated in these networks can provide rich information inputs especially for marketing and organizational communications, decision making, coaching, professional performance and engagement, etc. Furthermore, promoting a healthy emotional climate will boost for the community motivation and improvement of performance and commitment, which sometimes is difficult to sustain in such networks. Emotional conflicts between stakeholders may also influence the network, so the envisioned supervision system should trigger dynamic actions e.g. generating alerts or suggesting actions to be taken by the network coordinator, in order to reestablish the emotional equilibrium of the community.

\section{GloNet Network}

The GloNet network is an agile virtual enterprise environment for networks of SMEs involved in highly customized and service-enhanced products through end-to-end collaboration with customers and local suppliers (co-creation) in the solar park construction sector. The involved organizations range from mechanical and electrical to software product development enterprises in the area and the main focus is on the "Product lifecycle management".

A challenge in this network is the effective management of the interactions among participating organizations themselves and with the customers. Some participants' problems, that are not solvable with the technicalities of the project execution, might arise. These problems can include inter- and intra-organizational abilities, problems with the team, leadership, customers and the network as a whole. It might also include external factors such as the participating organization itself, the people that stand for 
the organization, etc. These are a result of conceived emotions from "environmental" perceptions. Considering these challenges, the manager's role of understanding and effectively managing the participating organizations by avoiding emotional conflict is a complex task.

The emotions supervision system might play an important role helping the network manager to take actions towards enhancing the quality of the interactions between the involved participating organizations. For this case, emotional solutions in the negotiation phases; during the operational phase and after the conclusion and deployment of the customized product are of extreme importance. Moreover, the inclusion of more precisely emotional information about the participant's competences (resorting to emotional intelligence methods and methodologies) would certainly help in the emotional management of the network.

\section{$6 \quad$ Related Research}

Collaborative Networks. The concept of Collaborative Network $(\mathrm{CN})$ has become stronger in recent years within the academic and industrial areas. It constitutes an effort to concretize and modernize the traditional concept of cooperation networks among companies referring essentially to shared work, which implies shared capabilities and resources, and the use of a 'network' to communicate and exchange information. Although several definitions can be found in the literature $[5,15,16]$, in this research work, the adopted definition is: " $A C N$ is a network consisting of a variety of entities (e.g. organizations, people, even intelligent machines) that are largely autonomous, geographically distributed, and heterogeneous in terms of their operating environment, culture, social capital and goals, but which decide to collaborate to better achieve common or compatible goals (e.g. problem solving, production, or innovation), and whose interactions are supported by computer networks" [4].

In the $\mathrm{CN}$ context, organizations interoperate and collaborate within Virtual Organizations (VO) and Virtual Organizations Breeding Environments (VBE) networks while being facilitated by computer networks, in order to achieve certain common or compatible goals, such as the acquisition of and response to larger, better, and more business opportunities. As a basic rule, supporting the dynamic/fluent formation of collaborative networks, such as in a short term consortium, requires its potential partners to be ready and prepared to jointly participate in such a collaboration environment. The foundation of this readiness should include reaching commonality agreements on aspects such as the interoperable infrastructure, operating rules, and cooperation. Any collaboration also requires that involved actors meet the required level of competency performance, and emotional equilibrium to be considered trustworthy by other partners. Therefore, the concepts of long-term strategic alliances have emerged as the necessary context for the effective creation of dynamic short term consortia. Moreover, with the consolidation of CNs as a new discipline more emphasis is being put on the elaboration of the theoretical foundation and reference models for the area. The ECOLEAD project designed the ARCON reference modeling framework for CNs [17]. The results from the ARCON reference modeling framework that are relevant for this work are mainly the behavioral endogenous elements or behavioral dimension. The behavioral dimension provides the context for integration and generalization of the various behavioral aspects 
of CNs. The principles of collaboration and rules of conduct (CN governance), where issues such as business process modeling, principles of trust, value systems, contracts negotiation and conflicts resolution, collaboration readiness, reward and incentives, among others can be found, are addressed with special focus in this dimension. It is also within this dimension that the emotions' modeling aspect proposed in this work fits.

Emotions. The meaning of emotion (animi motus in the Latin) is expressed in the term itself, that is, the idea of 'motion' in one's inward feelings and selfconsciousness. These inward motions of the 'soul' (psyche) signal and give rise to 'moods,' inner feelings and dispositions.

The concept of 'emotion' presents a particularly difficult problem. Even though the term is used very frequently, the question "What is an emotion?" rarely generates the same answer from different individuals and scientific researchers. This is due to the fact that emotions concern what is most intimate and important to human life and because some of their effects demand understanding. After some definitions analysis, it can be said that most theories hold that emotion is a complex entity with many components: physiological, cognition, sensory input, behavior correlates (e.g. expressions of emotion). In addition and common to all definitions is the undeniable value of emotion for people and the society. Emotions determine personal viability; prepare people for action; shape people's behavior; regulate social interactions; facilitate nonverbally communication; make life worth living by adding value to experience; allow people to respond flexibly to the environment (approaching good, avoiding bad) and have a central place in moral education and moral life through conscience, empathy, and many specific moral emotions such as shame, guilt, and remorse; inextricability linked to moral virtues.

Furthermore, the definition of emotion changes according to the point of view taken by the researcher, i.e., in accordance with several different disciplinary perspectives: biology, psychoanalysis, psychology (cognitive psychology, social psychology), sociology, organizational theory, and management, community and humanities studies. The most recent area focusing on the importance of humancomputer emotions, affective computing is a branch of the study and development or artificial intelligence that deals with the design of systems and devices that can recognize, interpret, and process human emotions (Fig. 3).

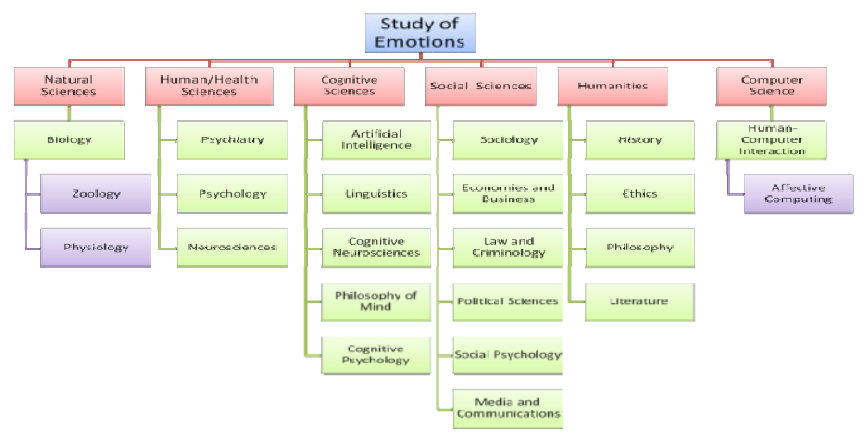

Fig. 3. Disciplinary perspectives of emotions' research 
Collective and Group-based Emotions. Collective emotions have been defined in a relatively general way as emotions that are shared by large numbers of individuals in a certain society [18] while group-based emotions are defined as emotions that are felt by individuals as a result of their membership in a certain group or society [19]. According to Bar-Tal, et al. [20], both concepts suggest that individuals may experience emotions, not necessarily in response to their personal life events, but also in reaction to collective or societal experiences in which only a part of the group members have taken part. But while the former concept suggests that group members may share the same emotions for a number of different reasons, the latter refers only to emotions that individuals experience as a result of identifying themselves with their fellow group members part [20].

Emotion labor or work. Arlie Hochschild [21], defined the concept of emotion work or emotion labor in terms of requirements or expectations at work to pretend emotions that were not genuinely felt (e.g. the forced niceness and inauthentic smile in a customer service interaction). She argued that emotion work alienates people from their emotions and creates a state of emotional dissonance between displayed emotions and actual feelings that over time, if not resolved, can have profound negative health impacts [22].

Mumby \& Putnam [23] define emotional labor as "the way individuals change or manage emotions to make them appropriate or consistent with a situation, a role, or an expected organizational behavior." Most professions have unwritten emotion display rules, e.g. nurses being empathetic and caring; police or debt collectors showing anger and impatience [24]. At times, emotional detachment may be a functional way of coping with a very stressful or difficult job, e.g. medical personnel dealing with death.

Emotional Intelligence. According to Goleman [25], emotional intelligence refers to "the capacity for recognizing our own feelings and those of others, for motivating ourselves, and for managing emotion in ourselves as well as in our relationships". It describes abilities distinct from, but complementary to, academic intelligence, the purely cognitive capacities measured by IQ. Many people who are 'book smart' but lack emotional intelligence end up working for people who have lower IQs than them but who excel in emotional intelligence skills. These two different kinds of intelligence - intellectual and emotional - express the activity of different parts of the brain.

A comprehensive theory of emotional intelligence was proposed in 1990 by two psychologists, Peter Salovey, at Yale, and John Mayer [26]. They proposed a model of emotional intelligence as: "a set of skills hypothesized to contribute to the accurate appraisal and expression of emotion in oneself and in others, the effective regulation of emotion in self and others, and the use of feelings to motivate, plan and achieve in one's life." Another pioneering model of emotional intelligence was proposed in the 1980s by Reuven Bar-On, an Israeli psychologist [27]. And in recent years several other theorists have proposed variations on the same idea.

Organizational Climate. "Organizational climate" is in sense the "personality" of the organization and includes relatively constant variables in a work environment. The common view is that organizational climate should be conceptualized as a set of global perceptions held by individuals about their objective organizational situations and interrelationships between them [28]. It is a psychological process in which 
organizational members perceive and characterize their environment in an attitudinal and value-based manner [29]. Furthermore, according to Voon, Hamali, and Tankau [30] a positive climate was found to improve the performance of organizations and teams.

Affective Computing. In computer science, affective computing is a branch of the study and development of artificial intelligence that deals with the design of systems and devices that can recognize, interpret, and process human emotions. It is an interdisciplinary field spanning computer sciences, psychology, and cognitive sciences. While the origins of the field may be traced as far back as to early philosophical enquiries into emotion, the more modern branch of computer science originated with Rosalind Picard's [14] paper on affective computing.

It is then a young field of research dealing with issues regarding emotions and computers that has as aim to give answers to questions such as what is the foundation of recognizing, understanding and expressing emotions (in and through computers)? And how can we build a computer able to feel?

Self-Healing Systems. Inspired by biology, self-healing has evolved as a property of IT systems that enables such systems to diagnose themselves and react to faults such that a satisfactory mode of operation is restored after the occurrence of a fault or a failure [31]. Self-healing is not confined to a certain class of IT systems but covers the broad span of computing from applications over system software to networking, from small embedded devices over personal computers to power grids.

Self-healing is also commonly used in psychological and social domains to resolve societal conflicts. It is defined as a process by which a party repairs the damage incurred over the course of (social) conflict, independent of the other party in the conflict [32]. In this perspective, healing aims at least partly, to reduce the negative aspects caused by the conflict [33]. According to Nets-Zehngut \& Bar-Tal [34] "selfhealing refers to healing on the level of the society considered as an entity, and implies that the healing takes place by the party itself, without collaborating with the other party in the conflict".

In this context, the adoption of these social concepts together with the self-healing systems of the IT software engineering domain will contribute to the advanced emotional self-regulation mechanisms that need to be developed and established in $\mathrm{CNs}$ in order to give dynamic responses to emotional changes maintaining in this way a healthy emotional network.

\section{$7 \quad$ Conclusions and Future Work}

This paper introduced an approach to develop and emotions-oriented supervision system aimed at assisting in the management of the participants' interactions and the emotional dynamics that affect the normal lifecycle of collaborative networks. Two validation scenario examples are briefly characterized and some potential emotional conflicts are identified for each case. The first phase of the work presented consisted on the identification and characterization of the principal requirements and future developments will comprise the elaboration of methods and mechanisms for the supervision system. 
Acknowledgments. This work has been supported by the Collaborative Networks and Distributed Industrial Systems Research Group of Uninova and partly by the GloNet and BRAID projects funded by the European Commission.

\section{References}

1. Camarinha-Matos, L.M., Afsarmanesh, H.: Collaborative Networks: A New Scientific Discipline. J. Intelligent Manufacturing 16(4-5), 439-452 (2005)

2. Abreu, A., Camarinha-Matos, L.M.: On the Role of Value Systems to Promote the Sustainability of Collaborative Environments. International Journal of Production Research 46(5), 1207-1229 (2008)

3. Bititci, U.S., et al.: Managing Synergy in Collaborative Enterprises. Production Palnning and Control 18(6), 454-465 (2007)

4. Camarinha-Matos, L.M., Afsarmanesh, H.: Collaborative Networks - Value Cration in a Knowledge society. In: PROLAMAT 2006. Springer, Shangai (2006)

5. Parung, J., Bititci, U.S.: A Metric for Collaborative Networks. Business Process Management Journal 14(5), 654-674 (2008)

6. Damasio, A.R.: Descarte's Error: Emotion, Reason, and the human Brain. Gosset/Putnam Press (1994)

7. Frijda, N.H.: The Emotion. Cambridge University Press, Cambridge (1986)

8. Johnson-Laird, P.N., Oatley, K.: Basic Emotions, rationality and folk theory. Cognition and Emotion 6, 201-223 (1992)

9. Mackie, D.M., Devos, T., Smith, E.R.: Intergroup Emotions: Explaining offensive actions in an intergroup context. Journal of Personality and Social Psychology 79, 602-616 (2000)

10. Camarinha-Matos, L.M., Afsarmanesh, H., Ferrada, F.: Collaborative Networks Approach to Active Ageing. In: AGEmap - Workshop on Roadmaps Towards a Strategic Research Agenda for ICT in Active Ageing, Munich, Germany (2010)

11. Msanjila, S., Afsarmanesh, H.: Trust Analysis and Assessment in Virtual Organizations Breeding Environments. International Journal of Production Research, 1253-1295 (2007)

12. Pouly, M., Monnier, F., Bertschi, D.: Success and Failure Factors of Collaborative Networks of SME. In: Camarinha-Matos, L.M., Afsarmanesh, H., Ortiz, A. (eds.) Collaborative Networks and Their Breeding Environments. Springer, Heidelberg (2005)

13. Camarinha-Matos, L.M., Macedo, P., Abreu, A.: Analysis of Core-Values Alignment in Collaborative Networks. In: Camarinha-Matos, L.M., Picard, W. (eds.) Pervasive Collaborative Networks, pp. 53-64. Springer, Boston (2008)

14. Picard, R.W.: Afective Computing. MIT Technical Report (1995)

15. Alves, A., et al.: High performance collaborative networks: a realistic innovation or just an academic desire? In: ERIMA 2007, Biarritz, France (2007)

16. Chituc, C.-M., Azevedo, A.L.: Multi-perspective challenges on collaborative newtorks business environment. In: Camarinha-Matos, L.M., Afsarmanesh, H., Ortiz, A. (eds.) Collaborative Networks and Breeding Environments, pp. 25-32. Springer, Heidelberg (2005)

17. Camarinha-Matos, L.M., Afsarmanesh, H. (eds.): Collaborative Networks: Reference Modeling. Springer, New York (2008)

18. Stephan, W.G., Stephan, C.W.: An integrated threat theory of prejudice. In: Oskamp, S. (ed.) Reducing Prejudice and Discrimination, pp. 225-246. Erlbaum, Hillsdale (2000) 
19. Smith, E.R.: Social identity and social emotions: Toward new conceptualization of prejudice. In: Mackie, D.M., Hamilton, D.L. (eds.) Affect, Cognition and Stereotyping: Interactive Processes in Group Perception, pp. 297-315. Academic Press, San Diego (1993)

20. Bar-Tal, D., Halperin, E., de Rivera, J.: Collective Emotions in Conflict Situations: Societal Implications. Journal of Social Issues 63(2), 441-460 (2007)

21. Hochschild, A.R.: The managed heart: Commercialization of human feeling. University of California Press, Berkeley (1983)

22. Tanner, K.J.: Emotion, Gender and the Sustainability of Communities. Journal of Community Informatics 1(2), 121-139 (2005)

23. Mumby, D.K., Putnam, L.L.: The politics of emotion: A feminist reading of bounded rationality. Academy of Management Review 17(3), 465-486 (1992)

24. Rafaeli, A., Sutton, R.I.: Emotional contrast strategies as means of social influence: Lessons from criminal interrogators and bill collectors. Academy of Management Journal 34(4), 749-775 (1991)

25. Goleman, D.: Working with emotional intelligence. Bloomsburry, London (1998)

26. Salovey, P., Mayer, J.D.: Emotional intelligence. Imagination, Cognition, and Personality (9), 185-211 (1990)

27. Bar-On, R.: The development of a concept of psychological well-being, Doctoral dissertation, Rhodes University, South Africa (1988); The precursor of the Bar-On model of emotional-social intelligence

28. Tyagi, P.K.: Perceived Organizational Climate and the Process of Salesperson Motivation. Journal of Marketing Research XIX, 240-254 (1982)

29. Verbeke, W., Volgering, M., Hessels, M.: Exploring the conceptual expansion within the field of organizational behavior: Organizational climate and organizational culture. Journal of Management Studies 25, 303-329 (1998)

30. Voon, B.H., Hamali, J., Taugkau, J.: Linking service climate to organizational performance: Evidence from Sarawak. International Journal of Business and Society 10(1), 18-26 (2009)

31. Rodosek, G.D., et al.: Self-Healing Systems: Foundations and Challenges. In: Andrzejak, A., et al. (eds.) Self-Healing and Self-Adaptive Systems. Schloss Dagstuhl - LeibnizZentrum fuer Informatik, Germany (2009)

32. Nets, R.: The collective self healing process of the aftermath of intractable conflicts (2005)

33. Frankel, E.: Repentance, psychotherapy and healing through a Jewish lens. The American Behavioral Scientist 41, 814-833 (1998)

34. Nets-Zehngut, R., Bar-Tal, D.: The intractable Israeli-Palestinian conflict and possible pathways to peace. In: Kuriansky, J. (ed.) Beyond Bullets and Bombs: Grassroots Peace Building Between Palestinians and Israelis, pp. 3-13. Praeger, Westport (2007) 